Удк 621.65

\title{
Алгоритм расчета демпфирующего колпака для поршневого насоса
}

\section{ХуиЮй Чжоу}

Одесский национальный политехнический университет, пр. Шевченко, 1, г. Одесса, 65044, Украина

$\bowtie$ e-mail: 170021121@qq.com

В статье представлена математическая модель колпака-гасителя колебаний давления в поршневых насосах и ее апробация на практических расчетах. Цель работы - получение теоретического обоснование обоснования для расчета колпака-гасителя колебаний давлений по заданным условиям. Показано, что известные два метода расчета колпаков-гасителей не удовлетворяют условиям практики. С помощью анализа передаточной функции колпака, как преобразователя (ослабителя) сигнала, получено соотношение, позволяющее рассчитать конструктивные характеристики колпака-гасителя по заданной степени снижения давления. Выполнены расчеты по условиям промышленных и энергетических насосов. Полученное выражение позволяет оценивать размеры колпака гасителя с учетом свойств газа и конструктивных условий на выходе из гасителя. Показано, что эфрективность колпака-гасителя, не зависит от режима работы насоса. Конструкция колпака-гасителя рассчитанная по предложенной методике обладает меньшей металлоемкостью, чем известные конструкции.

Ключевые слова: Колебания давления; Резонанс; Демпфирующий колпак; Передаточная функция; Гармонический сигнал.

\section{Алгоритм розрахунку демпфуючого ковпака для поршневого насоса}

\section{ХуиЮй Чжоу, О. В. Корольов}

Одеський національний політехнічний університет, пр. Шевченка, 1, м. Одеса, 65044, Україна

у статті представлена математична модель ковпака-гасителя коливань тиску в поршневих насосах і ії апробація на практичних розрахунках. Мета роботи - отримання теоретичного обгрунтування обгрунтування для розрахунку ковпака-гасителя коливань тисків по заданим умовам. Показано, що відомі два методи розрахунку ковпаків-гасителів не задовольняють умовам практики. За допомогою аналізу передавальної функції ковпака, як перетворювача (ослабителя) сигналу, отримано співвідношення, що дозволяє розрахувати конструктивні характеристики ковпака-гасителя по заданій ступеня зниження тиску. Виконано розрахунки за умовами промислових і енергетичних насосів. Отриманий вираз дозволяє оцінювати розміри ковпака-гасителя з урахуванням властивостей газу і конструктивних умов на виході з гасителя. Показано, що ефективність ковпака-гасителя, не залежить від режиму роботи насоса. Конструкція ковпака-гасителя розрахована за запропонованою методикою має меншу металоємність, ніж відомі конструкції.

Ключові слова: Коливання тиску; Резонанс; Демпфуючий ковпак; Передатна функція; Гармонічний сигнал.

DOI: http://dx.doi.org/10.15673/ret.v53i1.545

C The Author(s) 2017. This article is an open access publication

This work is licensed under the Creative Commons Attribution 4.0 International License (CC BY) http://creativecommons.org/licenses/by/4.0/

\section{1 Введение}

Целью настоящего исследования является теоретическое обоснование выбора оптимальных размеров колпака-гасителя колебаний давления в поршневых насосах. Расчет колпака-гасителя выполнен на анализе передаточной функции колпака, как преобразователя (ослабителя) сигнала. Полученное выражение позволяет оценивать размеры колпака-гасителя с учетом свойств газа и конструктивных условий на выходе из гасителя. 


\section{2 Анализ проблемы и постановка задачи ис- следования}

Проблемой поршневых насосов является повышенный уровень колебаний давления на выходе насоса, составляющие значения до $100 \%$ от номинального давления. Другой проблемой является невозможность пуска таких насосов без воздушных колпаков, позволяющих сглаживать колебания давления на выходе насоса и выравнивать крутящий момент на валу двигателя. Поэтому все поршневые насосы обязательно комплектуются воздушными колпаками, выступающими также как гасители колебаний давления.

Анализ литературы $[1,6,8-10,11]$ показывает, что теоретическое обоснование выбора оптимального размера камеры колпака основывается на двух основных направлениях. Первое - это расчеты колпака на максимальное пусковое давление и резонанс [7, 8, 12], получившие свое развитие в работах Н. Е. Жуковского о гидроударе [3]. В задачи этих расчетов входит, в частности, определение граничной частоты пропускания колпака, однако применительно к условиям, задающим определенный уровень снижения колебаний входного давления, эти расчеты не приспособлены. Такое положение определяется тем, что колпак рассматривается как сосредоточенный сжимаемый объем, и в целях упрощения расчетов рассматривается в основном его частотная характеристика. Второе направление специально предназначено для расчета элементов трубопровода как заградительного фильтра гармоническим сигналом. Теоретическое обоснование этих расчетов сводилось к проведению аналогий с электрическими системами и отождествлению рассматриваемого гасителя четырехполюснику с импедансным методом записи граничных условий $[1,2,4]$. Такая постановка позволяет решать задачи фильтрации или усиления гармонического сигнала в сложных гидравлических системах. Однако необходимость точного знания волнового сопротивления присоединенных участков, а также необходимость решения, как минимум, системы двух уравнений, не позволяет свести подобные расчеты к одной расчетной формуле. Такая необходимость часто возникает при вариациях конструктивного исполнения присоединительных к гасителю узлов, а также при частом повторении расчетов с разными технологическими условиями.

\section{3 Математическая модель колпака-гасителя}

Ниже представлена математическая модель колпака - гасителя гармонических пульсаций давления, основанная на анализе передаточной функции колпака, как преобразователя (ослабителя) сигнала [5].

Рассмотрим принципиальную схему подсоединения колпака к выходному патрубку насоса (рисунок 1). Примем, что 1/3 высоты колпака занята жидкостью и элементами подсоединения к напорной магистрали, а $2 / 3$ - газом.
Условие баланса массы газа в колпаке будет иметь вид

$$
\mathrm{V}_{\mathrm{k}} \frac{\mathrm{d} \rho \mathrm{r}}{\mathrm{dt}}=\mathrm{G}_{1}-\mathrm{G}_{2}
$$

где $\mathrm{V}_{\mathrm{k}}$ - объем занятый газом;

$\rho_{\mathrm{r}}$ - плотность газа;

$\mathrm{G}_{1}, \mathrm{G}_{2}$ - соответственно расход жидкости, входящей и выходящей из колпака.

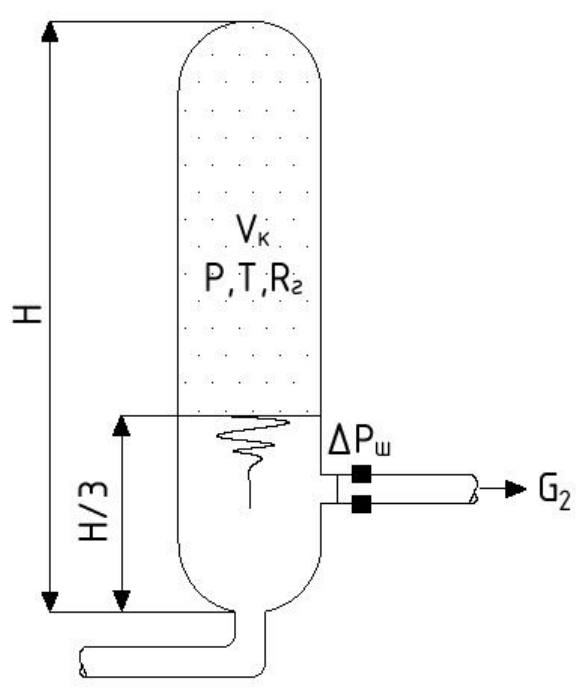

Рисунок 1 - Схема работы колпака-гасителя колебаний давления с дроссельной шайбой

Запишем выражение (1) в отклонениях от среднего значения:

$$
\mathrm{V}_{\mathrm{k}} \frac{\mathrm{d}\left(\rho_{\text {or }}+\delta \rho_{\mathrm{r}}\right)}{\mathrm{dt}}=\mathrm{G}_{01}+\delta \mathrm{G}_{1}-\mathrm{G}_{02}-\delta \mathrm{G}_{2}
$$

или, поскольку в стационарном режиме $\mathrm{G}_{01}=\mathrm{G}_{02}$, получим:

$$
\mathrm{V}_{\mathrm{k}} \frac{\mathrm{d} \delta \rho_{\mathrm{r}}}{\mathrm{dt}}=\delta \mathrm{G}_{1}-\delta \mathrm{G}_{2}
$$

где индекс " $\delta$ " показывает, что рассматривается отклонение от среднего значения.

Примем, что процесс сжатия-расширения газа в колпаке происходит по изотермическому закону:

$$
\mathrm{P}=\rho_{\mathrm{r}} \mathrm{R}_{\mathrm{r}} \mathrm{T}
$$

В отклонениях от среднего уравнение (3) примет вид:

$$
\begin{aligned}
& \delta \mathrm{P}=\delta \rho_{\mathrm{r}} \mathrm{R}_{\mathrm{r}} \mathrm{T} \\
& \delta \rho_{\mathrm{r}}=\delta \mathrm{P} / \mathrm{R}_{\mathrm{r}} \mathrm{T}
\end{aligned}
$$

Подставив (4) в (2):

$$
\frac{\mathrm{V}_{\mathrm{k}}}{\mathrm{R}_{\mathrm{r}} \mathrm{T}} \cdot \frac{\mathrm{d} \delta \rho}{\mathrm{dt}}=\delta \mathrm{G}_{1}-\delta \mathrm{G}_{1}-\delta \mathrm{G}_{2}
$$

Для исключения $\delta \mathrm{P}$ из уравнения (5) составим уравнение для течения жидкости через местное сопротивление на выходе из колпака:

$$
\mathrm{P}_{\mathrm{II}}=\mathrm{P}-\mathrm{P}_{1}=\mathrm{KG}_{2}^{2}
$$


где $\mathrm{K}$ - укрупненный коэффициент сопротивления. Записывая (6) в отклонениях, получим:

$$
\delta P-\delta P_{1}=K \cdot 2 G_{20} \delta G_{2}=\frac{2 \delta P_{\phi}}{G_{20}} \delta G_{2}
$$

Считая, что на выходе из колпака пульсации практически отсутствуют $\left(\delta \mathrm{P}_{1}=0\right)$, а расход равен номинальному $\left(\mathrm{G}_{20}+\mathrm{G}\right)$, приведем (7) к безразмерному виду:

$$
\delta P=\frac{2 \delta P_{\phi}}{P} \delta G_{2}
$$

Приведя (5) также к безразмерному виду, и подставив в него (8), получим уравнение динамики колпака-гасителя:

$$
\frac{2 \delta P_{\phi} V_{k}}{G R_{r} T} \cdot \frac{d \delta G_{2}}{d t}+\delta G_{2}=\delta G
$$

Для построения передаточной функции, преобразуем (9) в операторную форму, при этом обозначим

$$
\begin{gathered}
\frac{2 \delta P_{\grave{u}} V_{k}}{G R_{r} T}=\theta \\
s \theta \delta G_{2}+\delta G_{2}=\delta G_{1}
\end{gathered}
$$

Откуда непосредственно получаем:

$$
W(s)=\frac{\delta G_{2}}{\delta G_{1}}=\frac{1}{1+s \theta}=\frac{1}{1+s \varpi \theta},
$$

где $\omega$ - частота гармонического сигнала (колебания), проходящего через гаситель.

Для включения в расчет требуемых по технологии условий (снижение пульсаций расхода), представим эти условия в виде

$$
\left|\frac{\delta G_{2}}{\delta G_{1}}\right|=\sqrt{\operatorname{Re}^{2}}[W(s)]+I_{m}^{2}[W(s)]
$$

где $\left|\frac{\delta \mathrm{G}_{2}}{\delta \mathrm{G}_{1}}\right|$ - отношение амплитуд колебаний сигнала до и после гасителя

$$
\left(\delta G_{2} / \delta G_{1}=N\right) \text {. }
$$

С учетом (11), выражение (12) примет вид

$$
\left|\frac{\delta G_{2}}{\delta G_{1}}\right|=\frac{1}{\sqrt{1+\omega^{2} \theta^{2}}} .
$$

Полученное выражение позволяет оценивать размеры колпака-гасителя с учетом свойств газа и конструктивных условий на выходе из гасителя (в этом случае шайбование выхода вносит дополнительный гасящий эффект, т. к. повышается общее волновое сопротивление схемы). Выражение (10) получено при допущении, что газ в колпаке работает изотермически. Рассмотрим вариант, когда газ работает адиабатически, т. е. уравнение состояния имеет вид

$$
P=C \rho_{r}{ }^{n},
$$

где $\mathrm{C}$ - постоянная $\left(\mathrm{C}=\mathrm{P}_{0} / \rho_{0}^{n}\right)$;

$n$ - показатель адиабаты.
Последовательно логарифмируя и дифференцируя (15) представим его в отклонениях

$$
\delta P=\frac{n P}{\rho_{r}} \delta \rho r
$$

Подставим выражение (16) в выражение (12), получим

$$
\frac{\rho_{r} V_{k}}{n P} \cdot \frac{d \delta P}{d t}=\delta G_{1}-\delta G_{2} .
$$

Следуя далее аналогично изложенным выше выкладкам (6-8), получим выражение для постоянной времени $\theta_{\text {ад. }}$ При адиабатном процессе

$$
\theta_{\text {Ад }}=\frac{2 \Delta P_{u} \rho_{\Gamma} V_{k}}{n G P} .
$$

Подставляя (1.18) в (1.14), можно определить конструктивные размеры колпака при адиабатном процессе сжатия газа.

\section{4 Результаты расчета и практическое приме- нение}

Для расчетов размеров колпаков выражения (14, $10,18)$ удобнее представить в следующем виде:

$$
\left|\frac{\delta G_{2}}{\delta G_{1}}\right|=N \geq \frac{1}{\sqrt{1+\omega_{2}} \theta^{2}} .
$$

Откуда

$$
\theta \geq \sqrt{\left(1 / N^{2}-1\right)} \frac{1}{\omega} .
$$

Для условий изотермического процесса имеем

$$
\left(V_{k} \Delta P_{u}\right)_{\text {Из }} \geq \frac{1}{2} \sqrt{\left(\frac{1}{N_{2}}-1\right)} \frac{G R_{r} T}{\omega} .
$$

Для условий адиабатического процесса

$$
\left(V_{k} \Delta P_{u}\right)_{\text {Ад }} \geq \frac{1}{2} \sqrt{\left(\frac{1}{T^{2}}-1\right)} \frac{n G P}{\rho_{r} \omega} .
$$

В качестве примера, определим размеры гасителей для насоса 2 НСГ - 0,42/15.

1. Технические данные по насосу:

d - диаметр поршня - 25 мм;

Z - число цилиндров - 3 шт.;

$\mathrm{S}$ - ход поршня - 30 мм;

$\mathrm{d}_{\mathrm{o}}$ - диаметр вых. патрубка - 10 мм;

G - подача насоса (ном.) - 1500 кг/ч;

P - давление (макс.) - 15,0 МПа;

раб. среда - углекислота, азот;

t - раб. температуры $-20 . . .-40^{\circ} \mathrm{C} ;-196^{\circ} \mathrm{C}$.

2. Технические условия к расчету:

Снижение пульсаций расхода до 1 \%

$$
\left(\delta \mathrm{G}_{2} / \mathrm{G} \leq 0,01\right) \text {. }
$$


3. Определим относительное снижение пульсаций давления, требуемое в расчете,

$\frac{\delta \mathrm{G}_{1}}{\mathrm{G}_{1}}=\frac{\mathrm{Q}_{\text {мах }}-\mathrm{Q}_{\min }}{\mathrm{Q}_{\mathrm{cp}}}=0,14-$ относительные входные пульсации, по условиям работы насоса [6].

Тогда:

$$
\mathrm{N}=\frac{\delta \mathrm{G}_{2}}{\delta \mathrm{G}_{1}}=\frac{\delta \mathrm{G}_{2}}{\mathrm{G}} / \frac{\delta \mathrm{G}_{1}}{\mathrm{G}}=\frac{0,01}{0,14}=0,07 .
$$

4. Постоянная времени при заданном N и частоте $\omega$ :

$$
\begin{gathered}
\omega=356,5 \text { рад } / \mathrm{c} ; \\
\theta=0,084, \text { с. }
\end{gathered}
$$

5. Объем и длина гасителя (при $\varnothing_{\text {внут }}=56$ мм) при изотермическом и адиабатическом сжатии:

$$
\begin{aligned}
& \left(\Delta P_{u} V_{k}\right)_{T}{ }^{C O_{2}}=\frac{\theta}{2} \cdot \frac{G R_{r} T}{1}=\frac{0,084}{2} \cdot 0,439 \cdot 0,18895 \cdot 10^{3} \cdot 243=846,6 \text { Дж } \\
& \left(\Delta P_{u} V_{k}\right)_{A Д}{ }^{C O_{2}}=\frac{\theta}{2} \cdot \frac{n P G}{\rho}=\frac{0,084}{2} \cdot \frac{0,439 \cdot 150 \cdot 10^{5} \cdot 1,35}{1128,7}=330,8 \text { Дж } \\
& \left(\Delta P_{u} V_{k}\right)_{T}^{N_{2}}=\frac{\theta \cdot G R_{r} T}{2}=\frac{0,084}{2} \cdot 0,439 \cdot \frac{8,14 \cdot 10^{3}}{28} \cdot 77=412 \text { Дж} \\
& \left(\Delta P_{u} V_{k}\right)_{\text {АД }}^{N_{2}}=\frac{\theta}{2} \cdot \frac{n P G}{\rho}=0,439 \cdot \frac{150 \cdot 10^{5 \cdot, 4}}{846}=457,7 \text { Дж}
\end{aligned}
$$

Примем дополнительное местное сопротивление на выходе $\Delta \mathrm{P}_{ш}=1-3 \%\left(\mathrm{P}_{\mathrm{o}}\right)=0,5 \mathrm{MПа}$, тогда:

$$
\begin{array}{lc}
\left(V_{k}\right)_{T}{ }^{C O_{2}}=1,693 ; & \left(H_{k}\right)^{C O_{2}}{ }_{T}=0,688 M \\
\left(V_{k}\right)_{\text {АД }}{ }^{C O_{2}}=0,662 ; & \left(H_{k}\right)_{\text {АД }}{ }^{, M_{2}}=0,270 M \\
\left(V_{k}\right)_{T}{ }^{N_{2}}=0,803 ; & \left(H_{k}\right)_{T}{ }^{N_{2}}=0,326 M \\
\left(V_{k}\right)_{\text {АД }}{ }^{N_{2}}=0,901 ; & \left(H_{k}\right)_{\text {АД }}{ }^{N_{2}}=0,366 M
\end{array}
$$

Из двух результатов выбираем больший и окончательный размер гасителя:

$$
\begin{aligned}
& H_{k}^{C O_{2}}=\frac{3}{2}\left(H_{k}\right)_{T}{ }^{C O_{2}} \cong 1,0 M \\
& H_{k}^{N_{2}}=\frac{3}{2}\left(H_{k}\right)_{\text {АД }}^{{ }^{N_{2}}} \cong 0,55 M
\end{aligned}
$$

6. Диаметр шайбы для обеспечения перепада давления 0,5 МПа, при заданном расходе (1500 кг/ч):

$d_{\mathrm{CO}_{2}} \cong \sqrt{\frac{46}{n} \sqrt{\frac{1}{2 \Delta P_{u} \rho_{\text {Ж }}}}}=\sqrt{\frac{4 \cdot 0,439}{n}} \sqrt{\frac{1}{2 \cdot 5 \cdot 10^{5} \cdot 1128,7}}=4,96 \mathrm{MM} \approx 5 \mathrm{MM}$.

$d_{N_{2}} \cong \sqrt{\frac{4 \cdot 0,439}{n}} \cdot \frac{1}{\sqrt{2 \cdot 5 \cdot 10^{5} \cdot 846}}=4,38 \mathrm{MM} \approx 4 \mathrm{MM}$

При падении частоты работы поршневой группы, при неизменности размеров гасителя падает расход и давление нагнетания, при этом как следствие падает перепад давления по шайбе. Общая степень ослабления пульсаций давления гасителем будет определяться зависимостью (для адиабатического приближения):

$$
N \geq\left[1+\left(\frac{V_{k}}{n P \rho_{K_{u}} F_{u}^{2}} \cdot G \omega\right)^{2}\right]^{-1 / 2}
$$

где $\mathrm{F}_{\text {ш }}$ - площадь проходного сечения шайбы.

Таким образом, при падении расхода и частоты колебаний потока степень ослабления колебаний потока возрастает в пропорции от произведения $\mathrm{G \omega}$, т. е. в случае использования колпака не должно быть ослабления гашения пульсаций давления при изменении режима работы насоса.

Результаты экспериментального исследования разработанных в статье колпаков-гасителей представлены в [13].

\section{Выводы}

Анализ использования колпаков-гасителей показал необходимость разработки расчета колпаковгасителей с заданным ослаблением колебаний давления.

Разработана математическая модель, основанная на анализе передаточной функции колпака, как преобразователя (ослабителя) сигнала.

Полученное выражение позволяет оценивать размеры колпака гасителя с учетом свойств газа и конструктивных условий на выходе из гасителя, а также определять основные размеры системы по заданной степени ослабления колебаний давления.

Приведен пример расчета конструктивных размеров колпака-гасителя для промышленного насоса, показано, что степень ослабления колебаний не меняется с изменением режима работы насоса.

\section{Литература}

1. Владиславлев А.П. Трубопроводы поршневых компрессорных машин / Владиславлев А.П., Коробков А.А., Малышев В.А. и др. - М.: Машиностроение, 1972. $278 \mathrm{c}$.

2. Хачатурян С. А. Волновые процессы в компрессорных установках - М.: Машиностроение, 1983. - 222 с.

3. Жуковский Н. Е. О гидравлическом ударе в водопроводных трубах. Классики естествознания. - М.-Л.: ГИТТЛ, 1949. - 100 с.

4. Писаревский В. М. Гасители колебаний газа. - М.: Недра, 1986. - 120 с.

5. Морозов И. И. Устойчивость кипящих аппаратов / И. И. Морозов, В. А. Герлига. - М.: Атомиздат, 1969. $280 \mathrm{c}$

6. Чиняев И.А. Поршневые кривошипные насосы. - Л.: Машиностроение, 1983. - 176 с.

7. Чарный И.А. Неустановившееся движение реальной жидкости в трубах. - М-Л.: Гостехиздат, 1951. $223 \mathrm{c}$.

8. Фартуков В.А. Методы расчетов воздушных колпаков гасителей гидравлического удара // Изв. ВНИИГ, 1974. - т. 102. - с. 34

9. Korolyov O.V., Zhou Huiyu Dynamic damper pressure fluctuation in the pumping systems// Pratsi OPY, 2016. Issue 1(48). - p. 35-41. 
10. Stempniewski, M., Pompy. Warszawa, WNT, 1978. $560 \mathrm{I}$.

11. Фокс Д.А. Гидравлический анализ неустановившегося течения в трубопроводах. - М.: Энергоиздат, 1981. $-247 \mathrm{c}$.

12. Ситников Б.Т., Матвеев И.Б. Расчет и исследование предохранительных и переливных клапанов. - М.: Машиностроение, 1972. - 21-28 с.
13. Korolyov O.V., Zhou Huiyu, The study of pressure fluctuations in the pressure line of the pump and of the efficiency of the vibration absorbers // Pratsi OPY, 2016. Issue 3(50). - p.41-46.

Отримана в редакції 12.01.2017, прийнята до друку 07.02.2017

\title{
Algorithm for Calculation of Damping Cap for Piston Pump
}

\author{
HuiYu Zhou ${ }^{\varpi}$, O. V. Korolov
}

Odessa National Polytechnic University, 1 Shevchenko Ave., Odessa, 65044, Ukraine

$\triangle$ e-mail: 170021121@qq.com

\begin{abstract}
The paper presents a mathematical model of cap-damper of pressure oscillations in piston pumps and its approbation on practical calculations. The aim of the study is the theoretical justification of the rationale for the capdamper of pressure fluctuations calculation in the given conditions. It is shown that two previously known methods of cap-dampers calculation do not satisfy the conditions of the practice. By analyzing the transfer function of the cap as the converter (attenuator) of signal, the ratio was obtained, which allows to calculate the structural characteristics of the cap-damper on the desired degree of pressure reduction. Calculations under the terms of industrial and power pumps were carried out. The resulting expression allows you to evaluate the size of the cap-damper taking into consideration the properties of gas and structural conditions at the outlet of the absorber. It is shown that the effectiveness of the cap-damper does not depend on the pump operation mode. The design of the cap-damper calculated on the proposed method has a lower metal content than the conventional constructions.
\end{abstract}

Key words: Pressure Oscillations; Resonance; Cap-damper; Transfer Function; Harmonic Signal.

\section{References}

1. Vladislavlev, A.P. (1972) Truboprovody porshnievykh kompressornych mashyn. M, Mashynostroenie, $278 \mathrm{p}$.

2. Hachaturian, S.A. (1983) Volnovye processy v kompressornykh ustanovkakh. M, Mashynostroenie, $222 \mathrm{p}$.

3. Jukovskij, N.E. (1949) O gidravlicheskom udare v vodoprovodnykh trubakh. M-L, GITT, $100 \mathrm{p}$.

4. Pisarevskiy, V. M. (1986) Gasiteli kolebaniy gaza. M, Nedra, 120 p.

5. Morozov, I. I., Gerliga, V. A. (1969) Ustoychivost kipiaschih apparatov. M, Atomizdat, $280 \mathrm{p}$.

6. Chinyaev, I.A. (1983) Porshnevyie krivoshypnyie nasosy. L, Mashynostroenie, $176 \mathrm{p}$.

7. Charnyi, I.A. (1951) Neustanovivsheesya dvizhenie realnoy zhidkosti v trubah. M-L, Gostehizdat, $223 \mathrm{p}$.

8. Fartukov, V.A. (1974) Metodyi raschetov vozdushnyh kolpakov gasiteley gidravlicheskogo udara. Izv. VNIIG, 102, 34.
9. Korolyov O.V., Zhou HuiYu. (2016) Dynamic damper pressure fluctuation in the pumping systems, Pratsi $O P Y$, No. 1(48), 35-41.

10. Stempniewski, M., (1978) Pompy. Warszawa, WNT, 560.

11. Foks, D.A. (1981) Gidravlicheskiy analiz neustanovivshegosya techeniya $v$ truboprovodah. M, Energoizdat, $247 \mathrm{p}$.

12. Sitnikov, B.T., Matveev, I.B. (1972) Raschet i issledovanie predohranitelnyih i perelivnyih klapanov. M, Mashinostroenie, pp. 21-28.

13. Korolyov, O.V., Zhou, HuiYu. (2016). The study of pressure fluctuations in the pressure line of the pump and of the efficiency of the vibration absorbers. Pratsi $O P Y$, No. 3(50), 41-46. 